Article

\title{
Interferon Signaling in Chickens Plays a Crucial Role in Inhibiting Influenza Replication in DF1 Cells
}

\author{
Daniel S. Layton ${ }^{1, *,+}$, Kostlend Mara $^{1,+}{ }^{+}$, Meiling Dai ${ }^{1,+}{ }^{+}$, Luis Fernando Malaver-Ortega ${ }^{2}$, Tamara J. Gough ${ }^{1}$, \\ Kerri Bruce $\left.{ }^{1}{ }^{(}\right)$, Kristie A. Jenkins ${ }^{1}$ and Andrew G. D. Bean ${ }^{1}$
}

1 CSIRO Health and Biosecurity, Australian Centre for Disease Preparedness (ACDP), Geelong, VIC 3220, Australia; kostlend.mara@csiro.au (K.M.); meiling.dai@csiro.au (M.D.); tamara.gough@csiro.au (T.J.G.); Kerri.Bruce@csiro.au (K.B.); Kristie.Jenkins@csiro.au (K.A.J.); Andrew.Bean@csiro.au (A.G.D.B.)

2 Department of Biochemistry and Molecular Biology, Monash Biomedicine Discovery Institute, Clayton Campus, Monash University, Clayton, VIC 3800, Australia; luis.malaver-ortega@monash.edu

* Correspondence: daniel.layton@csiro.au; Tel.: +61-35-227-5433

+ These authors contributed equally.

check for updates

Citation: Layton, D.S.; Mara, K.; Dai, M.; Malaver-Ortega, L.F.; Gough, T.J.; Bruce, K.; Jenkins, K.A.; Bean, A.G.D. Interferon Signaling in Chickens Plays a Crucial Role in Inhibiting Influenza Replication in DF1 Cells. Microorganisms 2022, 10, 133.

https://doi.org/10.3390/

microorganisms10010133

Academic Editor: Kirill Sharshov

Received: 18 November 2021

Accepted: 30 December 2021

Published: 10 January 2022

Publisher's Note: MDPI stays neutral with regard to jurisdictional claims in published maps and institutional affiliations.

Copyright: (C) 2022 by the authors. Licensee MDPI, Basel, Switzerland. This article is an open access article distributed under the terms and conditions of the Creative Commons Attribution (CC BY) license (https:// creativecommons.org/licenses/by/ $4.0 /)$.

\begin{abstract}
Influenza A viruses (IAV) pose a constant threat to human and poultry health. Of particular interest are the infections caused by highly pathogenic avian influenza (HPAI) viruses, such as H5N1, which cause significant production issues. In response to influenza infection, cells activate immune mechanisms that lead to increased interferon (IFN) production. To investigate how alterations in the interferon signaling pathway affect the cellular response to infection in the chicken, we used CRISPR/Cas9 to generate a chicken cell line that lacks a functional the type I interferon receptor (IFNAR1). We then assessed viral infections with the WSN strain of influenza. Cells lacking a functional IFNAR1 receptor showed reduced expression of the interferon stimulated genes (ISG) such as Protein Kinase R $(P K R)$ and Myxovirus resistance $(M x)$ and were more susceptible to viral infection with WSN. We further investigated the role or IFNAR1 on low pathogenicity avian influenza (LPAI) strains (H7N9) and a HPAI strain (H5N1). Intriguingly, Ifnar ${ }^{-/}$cells appeared more resistant than WT cells when infected with HPAI virus, potentially indicating a different interaction between H5N1 and the IFN signaling pathway. Our findings support that ChIFNAR1 is a key component of the chicken IFN signaling pathway and these data add contributions to the field of host-avian pathogen interaction and innate immunity in chickens.
\end{abstract}

Keywords: IFNAR; interferons; innate immunity; interferon stimulated genes; antiviral response; influenza virus; viral infection

\section{Introduction}

Avian influenza A (AI) virus, as a zoonotic agent, represents a significant threat to public health and poultry production worldwide. Outbreaks in farmed chickens is of great concern, as many AI virus strains, such as $\mathrm{H} 5 \mathrm{~N} 1$ and $\mathrm{H} 7 \mathrm{~N} 9$ can be directly transmitted to humans and in some instances lead to high morbidity and mortality. Furthermore, the impact of such virus strains with pandemic potential on human health would be devastating, given the lack of specific vaccines and the emergence of drug resistant IAVs. Infection with LPAI viruses in poultry often results in subclinical infections or causes mild respiratory disorder accompanied with reduced egg production and low mortality. Conversely, infections caused by HPAI viruses can induce an acute disease with fatality as high as $100 \%$ in domestic poultry. Additionally, AI outbreaks result in a significant cost to the poultry industry, as the only containment measures are the quarantine or mass slaughtering of the birds. It is therefore critical to further our understanding of LPAI and HPAI to ascertain risk and develop new preventative measures. 
Innate immunity is the first line of protection against pathogen infection. A key component of innate antiviral immunity are IFNs, a class of cytokines with antiviral and immunomodulatory activity. Upon secretion, these molecules initiate signal transduction pathways leading to the up-regulation of the transcription of many genes, commonly named ISG, supporting an antiviral state in the surrounding cells [1]. In chickens, like mammals, there are three known classes of IFN, Type I, II and III. Type I IFNs were first identified by Isaacs and Lindeman [2] in influenza infected chicken embryos. Although these chicken IFNs have functional homologies with mammalian IFNs, chickens express a reduced numbers of Type I IFNs, in contrast to the different types of IFNs found in mammals [3]. Both IFN $\alpha$ and IFN $\beta$ bind to the chicken Type I IFN receptor complex (chIFNAR) which is comprised of two subunits, chIFNAR1 and chIFNAR2. Chicken IFN $\alpha$ and IFN $\beta$ share only $57 \%$ homology and the antiviral state induced by chIFN $\alpha$ has been shown to be stronger than that of chIFN $\beta$, which is likely attributed to the greater expression levels of downstream antiviral ISGs [4]. In a study by Qu et al., the anti-VSV activity of chIFN $\alpha$ was 100 -fold greater than the anti-VSV activity of chIFN $\beta$, based on the cytopathic effect inhibition assay on DF1 cells [5]. The same study also demonstrated that a differential expression of ISGs, such as $2^{\prime}, 5^{\prime}-\mathrm{OAS}$, PKR, IL-6, MHC-I, IFNAR-1 and IFNAR-2 was at the core of the different antiviral activities of both IFNs, with chIFN $\alpha$ showing a higher induction of these genes. Several studies, mainly in mouse models, have demonstrated that blocking the IFNAR receptor leads to high influenza viral loads, high mortality and decreased activation of ISGs such as PKR and Signal transducer and activator of transcription 1 (Stat1) [6]. Similarly, dendritic (DCs) and macrophages of Ifnar ${ }^{-/}$- mice infected with Lymphocytic Choriomeningitis Virus (LCMV) showed higher levels of viral infection than wild type (WT) mice, as well as higher levels of viral nucleoprotein (NP) [7]. In PBS-12SF chicken cells, shRNA knock-down of IFNAR1 resulted in higher production of influenza H1N1 and decreased expression of ISGs suggesting a limitation in the antiviral mechanisms controlled by IFNs [8].

Upon ligand binding, the IFNAR receptors are activated and able to recruit further effector molecules belonging the JAK/STAT family, leading to the formation of the IFNstimulated gene factor 3 (ISGF3) complex. This complex translocates into the nucleus, binds to IFN Stimulated Response Elements (ISRE) with consequential activation of the transcription of ISGs $[9,10]$. The ISGs play an important role in the antiviral response against the pathogen by acting at several stages of the virus replication cycle. Two of the most studied ISGs in chicken include the $M x$ and $P K R$ genes [11,12]. Mx is a key antiviral protein involved in blocking the early stages of viral replication. In mammals, there are two forms of the Mx protein, MxA and MxB. Interestingly, only MxA has been reported to be a potent inhibitor of influenza [13,14]. In birds, there is just one lineage of the $M x$ gene [3]. Although the avian Mx protein shares structural homologies with the human protein, its antiviral activity requires further analysis, with studies reporting contradictory results. A number of studies have demonstrated that a variant of Mx containing an asparagine (Asn) at position 613 (Mx-Asn 613) has an antiviral effect against VSV and Newcastle Disease Virus (NDV) [15-17], whereas other in vivo studies were unable to clearly demonstrate an antiviral effect against H7N1, H5N1 and H7N7 [18,19], or showed only moderate reduction in mortality in chickens following H5N2 infection [20]. Another well studied ISG in chickens is PKR [21], which is activated by dsRNA and responses to Toll-like receptor (TLR) mediated immune responses. Upon activation, it phosphorylates eukaryotic initiation factor 2 (eif2) with consequent inhibition of mRNA translation in infected cells. In vitro studies on chicken DF1 cells have shown upregulation of chicken PKR following IFN stimulation [5] confirming the involvement of $P K R$ in AI virus infection.

Whilst IFN signaling has been widely studied in mammals, it is clear that this pathway has yet to be fully elucidated in chickens. Thus, exploring the role of Type I IFN signaling may further elucidate the innate immune response involved in antiviral defense. In this study, we demonstrated that deletion of the Type I IFN response, by blocking the Type I transduction signal through deletion of the chIfnar1 gene, prevented activation by known 
ISGs. Furthermore, we showed that chIFNAR1 ${ }^{-/-}$cells were more susceptible to virus infection, potentially due to a shorter virus cycle. Interestingly, decreased viral infection was found in chIFNAR1 ${ }^{-/-}$cells upon infection with HPAI H5N1 compared to WT cells, suggesting an important functional interaction with HPAI and IFN signaling. These findings may have implications for our understanding of the innate immunity in chickens and the antiviral response that is activated following influenza infections.

\section{Materials and Methods}

\subsection{Cell Culture and Transfection Procedure}

The chicken fibroblast cell line DF1 [22] (American Type Culture Collection number: CRL-12203) was provided by the tissue culture laboratory at the CSIRO Australian Centre for Disease Preparedness. The DF1 cells were maintained in Dulbecco's Modified Eagle's Medium supplemented with 10\% fetal calf serum (FCS), $2 \mathrm{mM}$ L-glutamine, $10 \mathrm{mM}$ HEPES, $1.5 \%(w / v)$ sodium bicarbonate, $100 \mathrm{U} / \mathrm{mL}$ penicillin and $100 \mu \mathrm{g} / \mathrm{mL}$ streptomycin and incubated at $37^{\circ} \mathrm{C}$ in a $5 \% \mathrm{CO}_{2} / 95 \%$ air atmosphere.

\subsection{Virus Strains}

The Influenza virus strains used in this study LPAI Influenza A/WSN/33 (H1N1); HPAI Influenza A/Vietnam/1203/2004 (H5N1); Influenza A/Anhui/1/2013 (H7N9) were propagated by allantoic cavity inoculation of 9-11-days of embryogenesis specific-pathogenfree (SPF) embryonated chicken eggs. The virus stock was titrated in chicken eggs and the $50 \%$ egg infectious dose (EID50)/mL was calculated according to Reed and Muench [23]. All in vitro work involving Influenza A/WSN/33 (H1N1) was conducted within BSL-2 facilities at ACDP. All experiments with infectious HPAI Influenza A/Vietnam/1203/2004 (H5N1) and Influenza A/Anhui/1/2013 (H7N9) were conducted under BSL3 enhanced containment at ACDP approved by the CSIRO ACDP Institutional Biosafety Committee with Approval \# PARA 2019/003.

Virus titrations were performed on Madin-Darby canine kidney (MDCK; ATCC \#CCL34) in flat bottom 96-well plates in DMEM supplemented with $0.5 \%$ bovine serum albumin and $1 \mu \mathrm{g} / \mathrm{mL}$ of TPCK trypsin. Cultures were carried out for 5 days and cytopathic effects were determined and TCID50 calculated.

\subsection{Protein Modelling}

The structure of chIFNAR1 was predicted by retrieving the amino acid sequence of both chicken (G. gallus AAU93528.1) and human (H. sapiens AAT49100.1) IFNAR1 from the Genbank database. The protein sequences were modelled using the Phyre2 webserver to create predicted protein structures for both the chicken and human IFNAR1 receptor [24].

\subsection{CRISPR Guide Selection and Plasmid Construction}

We used the RNA-guided Cas9 nuclease from the microbial clustered regularly interspaced short palindromic repeats (CRISPR/Cas9) system, to produce a dual double-strand break (DSB) by duplexing constructs encoding two guides RNA (sgRNA) as previously reported (Ran et al., 2013). Briefly, two sgRNA (GCCGCGTGCGCAGTCGTCAGAGG, left hand and AGCACCGGGACACCACGACCAGG, right hand); were cloned into to the pSpCas9(BB)-2A-GFP (Plasmid ID: 48138; ADDGENE) and transfected into the continuous chicken embryo fibroblast cell line (DF1). The two sgRNA acted together to produce a deletion in the chicken IFN (alpha, beta and omega) receptor 1, chIfnar1 gene (Gene ID: 395665). The expected deletion was 97 base pairs (bp). Cells Transfected by Lipofectamine ${ }^{\mathrm{TM}}$ 2000, were sorted using a BD FACS Aria II cell sorter based on their GFP expression. A second round of sorting was performed to obtain single clones for further expansion and genomic DNA (gDNA) PCR screening. 


\subsection{Genomic DNA Isolation and PCR Analysis of ChIfnar1 Gene}

The identification of chIfnar1 knockout (KO) cells was simplified to a quick gDNA PCR screening on clonogenic isolations of the cell lines after sorting. Genomic DNA from transfected and WT cells was extracted using DNeasy Blood \& Tissue Kit (Qiagen, VIC, Australia) as per the manufacturer's instruction. PCR was performed using GoTaq Flexi DNA Polymerase (Promega) following manufacturer's instructions. Primers for screening were: Forward $5^{\prime}$-CGGCCACCCAAACCTTAGAA- $3^{\prime}$ and reverse $5^{\prime}$-CCATCTCGCAGCAGTTGTCT- $3^{\prime}$. to confirm the identity and extent of the deletion, amplicons were excised, purified from the gel (The Wizard ${ }^{\circledR \circledast}$ SV Gel and PCR Clean-Up System) and cloned into the pGEMt-Easy vector (Promega) for sequencing and analysis at Micromon Genomics sequencing facility (Monash University, Clayton, VIC, Australia).

\subsection{Flow Cytometry and Cell Sorting}

Influenza infected and uninfected cells were harvested and permeabilized with $\mathrm{BD}$ Fix/Perm solution (BD Biosciences, San Diego, CA, USA). The cells were then washed with Perm/Wash buffer and incubated with the primary antibody mouse anti-Influenza A Nucleoprotein antibody (Bio-Rad) for an hour at room temperature. The cells were again washed in Perm/Wash buffer and incubated with goat anti-mouse Alexa Fluor 488 (Life Technologies, Carlsbad, CA, USA) secondary antibody for an hour at room temperature. The cells were washed in Perm/Wash buffer, resuspended in FACS buffer (PBS, $4 \%$ FCS, $0.01 \%$ Sodium Azide) and analyzed using an LSR II flow cytometer (Becton-Dickinson, Franklin Lakes, NJ, USA). The data were processed using BD FACSDiva software (BectonDickinson, Franklin Lakes, NJ, USA) and analyzed with Flowlogic software (Version 7.2.1, Inivai Technologies, Mentone, VIC, Australia).

\subsection{Quantitative Real Time PCR ( $q R T-P C R)$}

The RNA from DF1 cells was extracted using the RNeasy Plus kit (QIAGEN) followed by cDNA synthesis using the SuperSript III One-step RT-PCR system kit (Life Technologies, Carlsbad, CA, USA) according to manufacturer's instructions. The relative quantitation of gene expression was determined using a Real-Time PCR System and the comparative threshold cycle $(\mathrm{Ct})$ method was used to show change in gene expression according to manufacturer's instructions (Applied Biosystems, Foster City, CA, USA). IFNAR1 transcription levels were analyzed using the Gg03338945_m1 assay (Cat\# 4351372), primer/probe sequences were undisclosed. Relative gene expression was calculated using the mean values obtained from $\Delta \Delta \mathrm{Ct}$ relative to the endogenous control housekeeper gene glyceraldehyde-3-phosphate-dehydrogenase (GADPH). The qRT-PCR primers and probes (Table 1) used for the detection of chicken IFN $\alpha, M x$ and $P K R$ genes have been previously described $[25,26]$. 
Table 1. PCR and qRT-PCR primers and probes sequences.

\begin{tabular}{|c|c|c|c|}
\hline Target Gene & Primer/Probe & Sequence $\left(5^{\prime}-3^{\prime}\right)$ & Accession No. \\
\hline \multirow{3}{*}{ GAPDH } & $\mathrm{F}$ & CCCCAATGTCTCTGTTGTTGAC & \multirow{3}{*}{ AF047874 } \\
\hline & $\mathrm{R}$ & CAGCCTTCACTACCСTCTTGAT & \\
\hline & Probe & CTTGGCTGGTTTCTCC & \\
\hline \multirow{3}{*}{$\mathrm{IFN} \alpha$} & $\mathrm{F}$ & GGACATGGCTCCCACACTAC & \multirow{3}{*}{ X92476 } \\
\hline & $\mathrm{R}$ & TCCAGGATGGTGTCGTTGAAG & \\
\hline & Probe & CAGCGCGTCTTGCTC & \\
\hline \multirow{3}{*}{ PKR } & $\mathrm{F}$ & GCAGAAGTAAGAGTGAGGCAAATGA & \multirow{3}{*}{ HQO14737 } \\
\hline & $\mathrm{R}$ & GCCACCTTTACCAATAGGCTCTAT & \\
\hline & Probe & CTGTGGATGAAAGGTTTC & \\
\hline \multirow{3}{*}{$\mathrm{Mx}$} & $\mathrm{F}$ & GTCCAAGAGGCTGAATAACAGAGAA & \multirow{3}{*}{ CR 389077} \\
\hline & $\mathrm{R}$ & GGTCGGATCTTTCTGTCATATTGGT & \\
\hline & Probe & CTGCTGCCTCATCCTT & \\
\hline \multirow{2}{*}{ IFNAR1 } & $\mathrm{F}$ & CGGCCACCCAAACCTTAGAA & \multirow{2}{*}{ Gene ID: 395665} \\
\hline & $\mathrm{R}$ & CCATCTCGCAGCAGTTGTCT & \\
\hline
\end{tabular}

\subsection{Statistical Analyses}

To determine the significant difference between uninfected and IFN $\alpha / \mathrm{IAV}$ infected cells, a one-way ANOVA with multi-comparisons analysis was performed. Instead, to determine the significant difference between the percentage of infected vs. non-infected cells and the Mean Fluorescence Intensity (MFI) a Ratio paired $t$-test was performed. Alpha for all tests was set at 0.05 and results were considered significant if $p$ values of less than 0.05 were obtained. Error bars represent the standard error of the mean (SEM).

\section{Results}

\subsection{Characterization of the Chicken Ifnar1}

While the structure of human IFNAR1 has already been characterized, little information is available regarding the structure and function of chIFNAR1. Structural analyses between chicken and human IFNAR1 receptor revealed structural homology between the two proteins. Similar to the human counterpart, chIFNAR1 structure is composed of three main structural domains, an ectodomain (ECD), a transmembrane and a cytosolic domain. This homology in structural architecture between chicken and human IFNAR1, suggests a similar function of the receptor. Furthermore, we evaluated the phylogenetic relationship between IFNAR1 amino acid sequences from multiple species. Based on the clustering patterns and sequence homologies, the phylogenetic analyses clustered the proteins in three groups. Although chIFNAR1 has functional homologies with its mammalian counterpart (human, chimpanzee, pig, bat and mouse), it was clustered together with the other bird species (duck and owl) suggesting an evolutionary divergence for avian species. The third group was composed by a reptile (snake). This avian divergence may suggest an important functional difference (Figure 1B). In mammals, the binding of the IFNs to their receptor initiates a complex signal transduction pathway which results in the activation of IFN regulated/stimulated genes. One of these ISGs, which has been widely studied is $M x$. In order to confirm whether this was the case in chickens, DF1 wild-type (WT) cells were stimulated with IFN $\alpha$ for $6 \mathrm{~h}$ and transcription levels of $M x$ were measured by qRT-PCR (Figure 1C). The results showed that stimulated cells had significantly higher levels of $M x$ transcripts compared to unstimulated cells, indicating a mechanism similar to that found in mammals. 
A

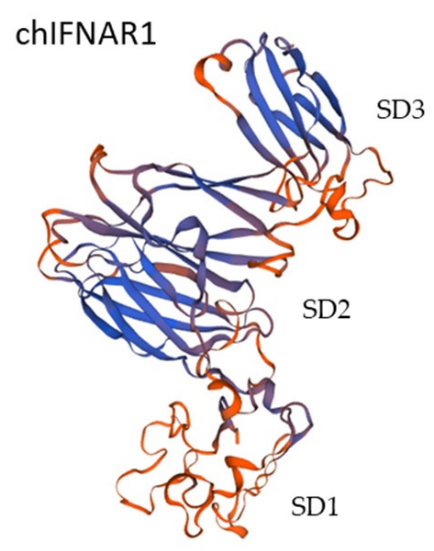

B

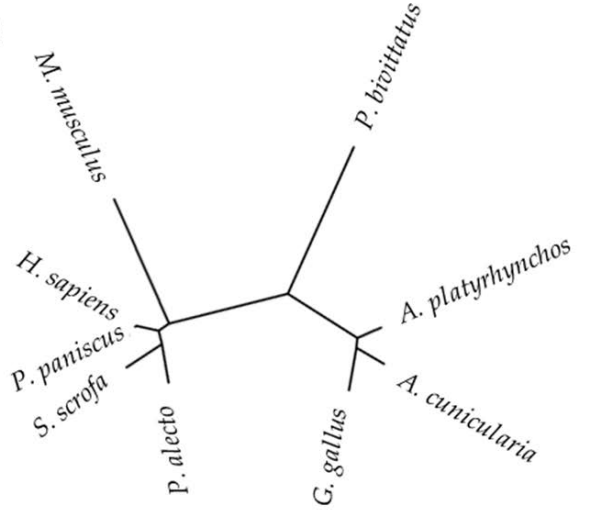

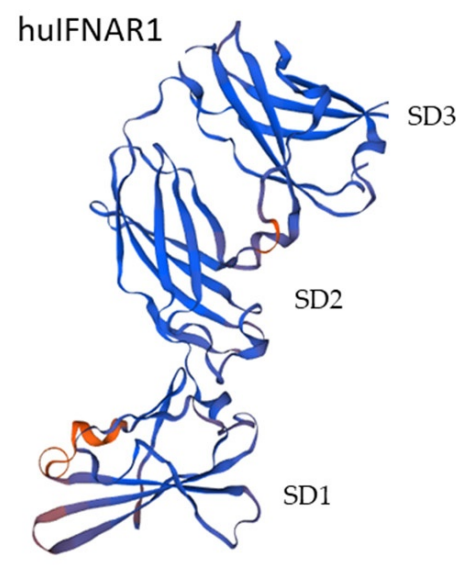

C

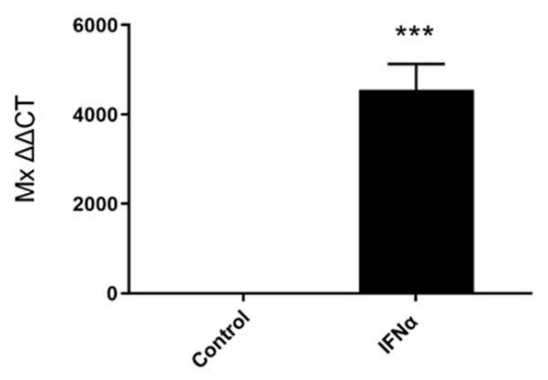

Figure 1. Chicken IFNAR1 is structurally and functionally similar to human. (A) Predicted tertiary structure of the ECD of chIFNAR1 compared to hIFNAR1. In blue are indicated $\beta$-sheets, in orange the coils. The four fibronectin type III (FNIII)-like subdomains are named SD1-SD4. (B) Phylogenetic relationship between IFNAR1 protein sequences from different species. The Accession Numbers of each IFNAR1 sequence were retrieved from the ensemble database. Chicken (G. gallus AAU93528.1), human (H. sapiens AAT49100.1), mouse (M. musculus AAH43935.1), duck (A. platyrhynchos XP_021124082.1), pig (S. scrofa CAJ76278.1), bat (P. alecto XP_006921038.1), snake (P. bivittatus XP_007442083.1), chimp (P. paniscus XP_003824035.1) and owl (A. cunicularia XP_026702394.1). (C) IFN $\alpha$ stimulates Mx through IFNAR1 on Chicken DF1 cells. Chicken DF1 cells were stimulated with ChIFN $\alpha$. Cells were harvested for RNA isolation, and cDNA preparation. Expression of Mx in control (white) and IFN $\alpha$ (black) stimulated cells was assayed by real-time PCR. Data were shown as mean \pm SEM $(\mathrm{n}=3)$. A value of $p<0.05$ was considered statistically significant. Data marked with $(* * *)$ indicate $p<0.001$.

\subsection{CRISPR Knock out of the ChIfnar1}

In order to study the role of the chIfnar1 gene in Type I IFN signal transduction we generated a mutant DF1 cell line by editing the genetic sequence of the gene using the CRISPR knock out technique. For this purpose, we designed two sgRNAs, sgRNA-1 and sgRNA-2, both targeting the first exon of the gene (Figure 2A). The transfection of WT DF1 cells generated a deletion of $97 \mathrm{bp}$ in the cellular genome as confirmed by sequencing the genomic DNA of the chIfnar1 locus (Figure 2D). The cells were cloned, and genomic characterization of these clones demonstrated that some of the clones showed a deletion in just one of the alleles, thus generating a monoallelic chIfnar $1^{+/}$clone, whereas other clones showed a deletion in both alleles, generating biallelic chIfnar $1^{-/}$clone. The genomic profile of the clones was further confirmed by PCR analyses and genomic sequencing. It indicated the presence of a double band (WT and deletion bands) for the monoallelic cells, and a single band corresponding to the deletion of a $97 \mathrm{bp}$ fragment in both alle- 
les for the biallelic cells, respectively (Figure 2B). Furthermore, we determined that the deletion also impacted the transcription levels of the gene. The transcription levels for WT, chIfnar $1^{+/-}$(monoallelic) and chIfnar $1^{-/-}$(biallelic) clones were assessed by qRT-PCR analyses (Figure 2C). As expected, both monoallelic and biallelic chIfnar1 mutants showed a significant reduction of their transcripts compared to the WT DF1 cells. Interestingly, the biallelic clone demonstrated a strong reduction, more than 4-fold compared to WT cells, but not complete ablation of the transcript, indicating that a small amount of transcript containing the deletion is still produced.

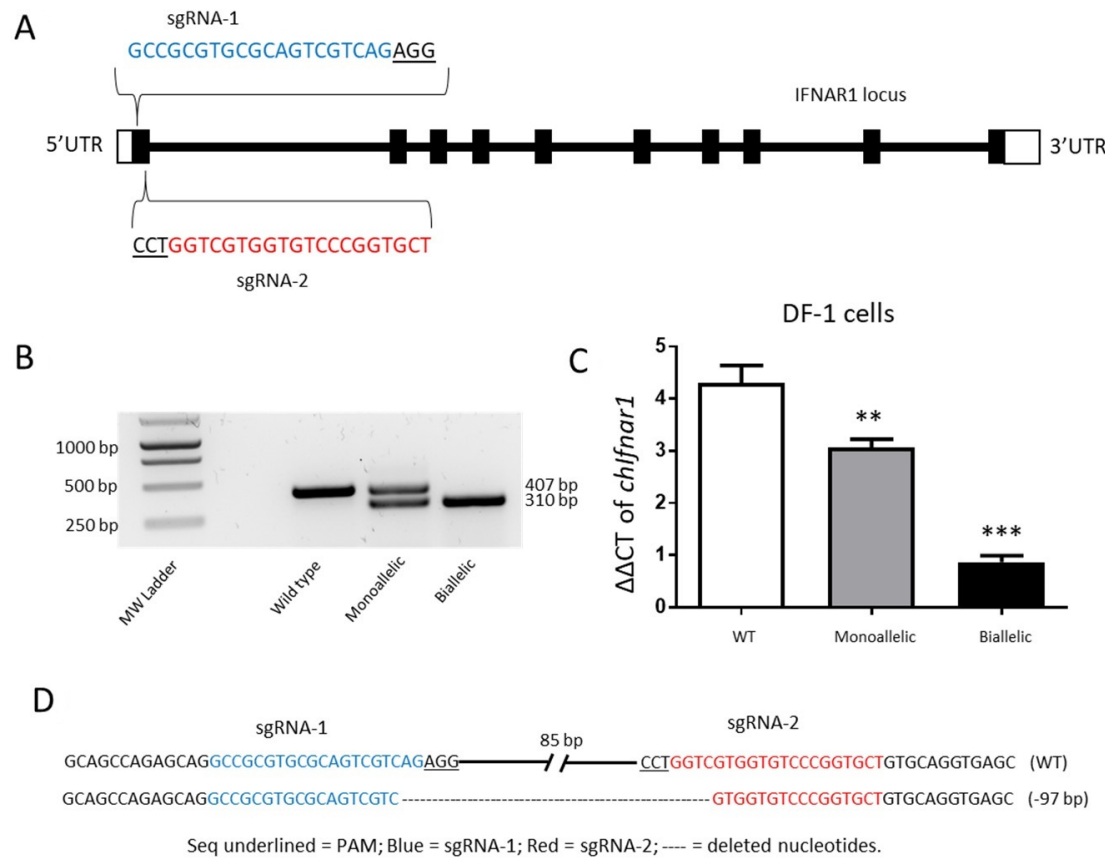

Figure 2. CRISPR/Cas9 mediated deletion of chIfnar1 Exon1. (A) chIfnar1 structure showing sgRNA targeting. Black boxes $=$ exons, white boxes $=5^{\prime}$ UTR and $3^{\prime}$ UTR. The introns are shown in dark bold horizontal lines connecting the exons. sgRNA sequences and location are shown with PAM sequence is underlined. (B) Gel electrophoresis image of Wild type, Monoallelic and Biallelic deletions detected by PCR. (C) Expression of chIfnar1 in WT (white), Monoallelic (grey) and Biallelic (black) assayed by real-time PCR. Data were shown as mean \pm SEM $(\mathrm{n}=3)$. Data marked $\left.{ }^{* *}\right)$ indicates $p<0.01$, and ${ }^{* * *}$ ) indicates $p<0.001$. (D) Sanger sequencing of the IFNAR locus confirmed the deletion. Sequences in blue represents the sgRNA-1 and in red the sgRNA-2 sequences, respectively. - = deleted nucleotides.

\subsection{Presence and Impact of ChIfnar1 on DF1 Cells}

Type I IFNs are activated following influenza infection and the role of IFNAR1 in the Type I IFN response to viral infection has been described in detail for mammalian hosts, but such mechanism is still unclear in the chicken. In order to study the impact of the IFNAR1 deletion in the chicken, we investigated the effects of IFN $\alpha$ on the activation of two ISGs, $M x$ and PKR [27]. Chicken DF1 WT and chIfnar1 ${ }^{-} /^{-}$mutant cells were stimulated with recombinant ChIFN $\alpha$ and infected with Influenza A/ WSN/ 33 (H1N1), either alone or in combination. The transcript levels of $M x$ (Figure 3A) and PKR (Figure 3B) were analyzed at 6 and $48 \mathrm{~h}$ after ChIFN $\alpha$ stimulation and/or viral infection. Quantitative RT-PCR analyses showed that both $M x$ and $P K R$ genes were significantly upregulated in both WT and chIfnar $1^{+/-}$monoallelic cells upon stimulation with IFN $\alpha$ alone or IFN $\alpha$ and IAV combined after $6 \mathrm{~h}$ of stimulation. Following $48 \mathrm{~h}$ stimulation/infection, significant increases in levels of $M x$ and PKR expression were detected only when IFN $\alpha$ and influenza were both present, in both WT and monoallelic cells. Results also revealed that the levels of gene transcription for both ISGs are higher at $6 \mathrm{~h}$ and tend to decrease at $48 \mathrm{~h}$, suggesting that $M x$ and $P K R$ are 
active in the early stages of infection. While the effect of IFN $\alpha$ stimulation, as suspected, was observed just in the first $6 \mathrm{~h}$, results showed that WSN alone was not able to stimulate the two ISG expressions at either time points. However, $M x$ and $P K R$ gene expression was observed at both time points following a combined stimulation with IFN $\alpha$ and WSN. In the case of the $48 \mathrm{~h}$ timepoint this indicates a possible synergetic effect of the two agents.

A $M x$

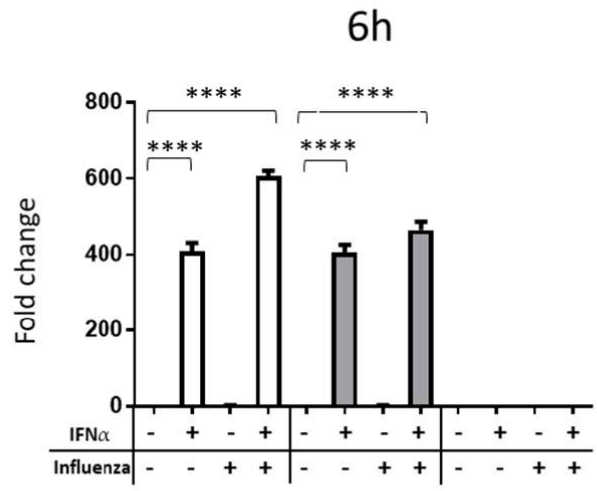

B PKR

$6 \mathrm{~h}$

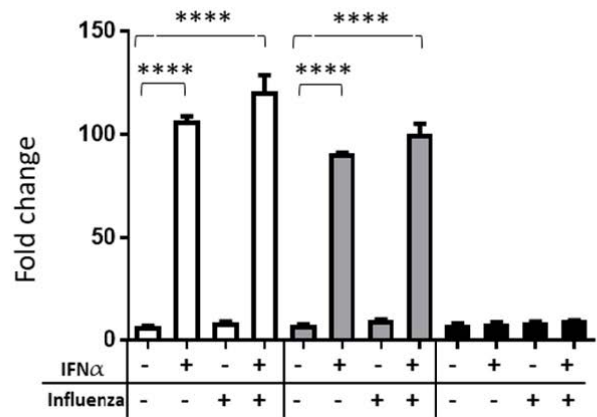

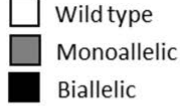

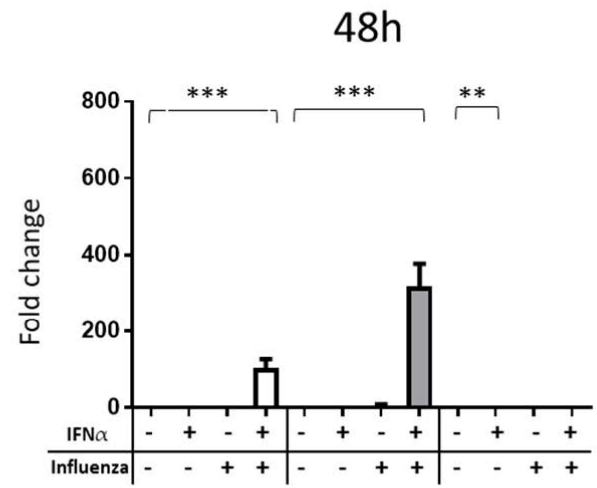

Figure 3. Functional characterisation of chIfnar1 deletion. After DF1 cells were stimulated with ChIFN $\alpha$, the cells were harvested at the indicated time points for RNA extraction and cDNA preparation. The transcriptional levels of (A) $M x$ and (B) PKR were measured by real time PCR following IFN $\alpha \pm$ influenza infections in WT (white), Monoallelic (grey) and Biallelic (black) cells at $6 \mathrm{~h}$ and $48 \mathrm{~h}$ post stimulation. Data were shown as mean \pm SEM $(n=3)$. The one-way ANOVA with multicomparisons statistical test was used to compare the differences in relative mRNA levels between ChIFN $\alpha \pm$ influenza treatments and DF1 controls at the same time points. A value of $p<0.05$ was considered statistically significant. Data marked with $\left(^{* *}\right)$ indicates $p<0.01$, and $(* * *)$ indicates $p<0.001$ and $\left(^{* * * *}\right)$ indicates $p<0.0001$.

Neither IFN $\alpha$ nor influenza infection were able to stimulate $M x$ or $P K R$ in the biallelic chIfnar $1^{-/-}$, which is in agreement with the observation in mammals [28], indicating that activation of $M x$ and PKR in chickens is stimulated by Type I IFNs through activation of the Type I IFN receptor IFNAR1. Cells with the IFNAR1 receptor deleted are unable to transduce the IFN signal and activate antiviral effector genes. This would suggest that it is likely that the chIfnar $1^{-/}-\mathrm{KO}$ cells had no remaining functional protein.

Interestingly, the basal level PKR expression dropped following influenza infection in biallelic chIfnar1-/- knock-out cells. 


\subsection{ChIfnar1 Knock out Impacts the Growth of the Influenza Virus WSN}

To evaluate the impact of the chIfnar1 knock-out on the susceptibility to IAV infection, we performed a time course infection experiment on both WT and chIfnar $1^{-1-}$ cells. The WSN influenza strain was chosen as DF1 cells are both susceptible to WSN in the absence of trypsin, and present show a cytopathic effect (CPE). Following infection with Influenza A/WSN at an MOI $=1$, supernatant containing virus was collected after 2, 4, 8, 12, 16 and $24 \mathrm{~h}$ post infection (hpi) and virus titre was quantified by TCID50 assay (Figure 4). Our data showed that the virus was able to replicate faster as indicated by significantly higher titres in chIfnar $1^{-/-}$cells compared to WT at $8 \mathrm{~h}$ and $12 \mathrm{~h}$ post infection (Figure 4 ). The virus released in the supernatant of the $\mathrm{KO}$ cells reached its highest concentration at $18 \mathrm{hpi}$, compared to $24 \mathrm{hpi}$ in the WT cells (Figure 4), suggesting a shorter virus replication cycle in chIfnar $1^{-/-}$cells than in WT cells.

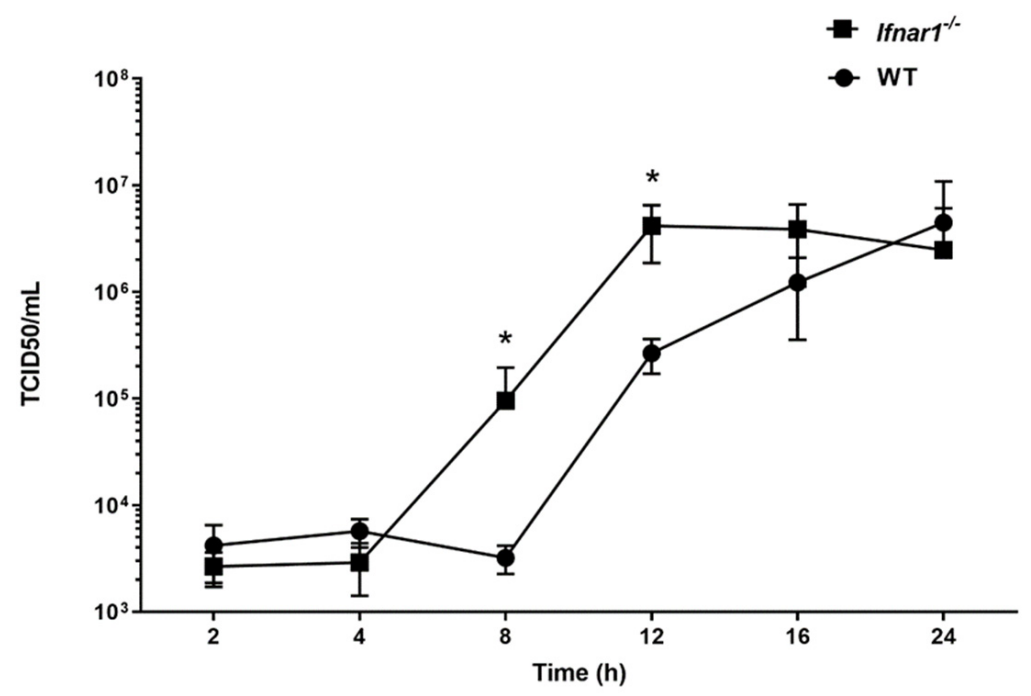

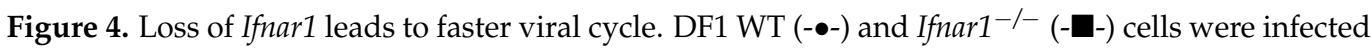
with Influenza A/WSN/33 (H1N1). At the indicated time post infection, supernatant containing virus was harvested and viral load was measured by TCID50 assay on MDCK cells. Data were shown as mean $\pm \operatorname{SEM}(\mathrm{n}=3)$. Data marked with $\left(^{*}\right)$ indicate $p<0.05$.

In addition, to validate the kinetics results, we measured intracellular viral nucleoprotein (NP) by flow cytometry (FACS). WT and chIfnar $1^{-/-}$cells were infected with WSN at different MOI (0.1 and 0.4) for 6, 24 and $48 \mathrm{~h}$. Analysis showed higher levels of intracellular viral protein, at $48 \mathrm{hpi}$ compared to the earlier time points (Figure 5A,C) for both cell types. Additionally, a higher percentage of infected cells was observed when cells were infected with a higher MOI $=0.4$. However, the percentage of infected cells in the chIfnar $1^{-1-}$ is higher than in the WT at each time point and different MOIs. These results suggest that chIfnar $1^{-1-}$ cells are more susceptible to IAV infection than WT.

Mean Fluorescence Intensity (MFI) of both infected WT and mutant cells at each infection time point and infection conditions were then measured to investigate whether the higher susceptibility of chIfnar $1^{-/}$cells is related to the increased presence of viral particles within the cells. No significant difference was observed between WT and chIfnar ${ }^{-1-}$ (Figure 5B), suggesting NP content was unaffected. 

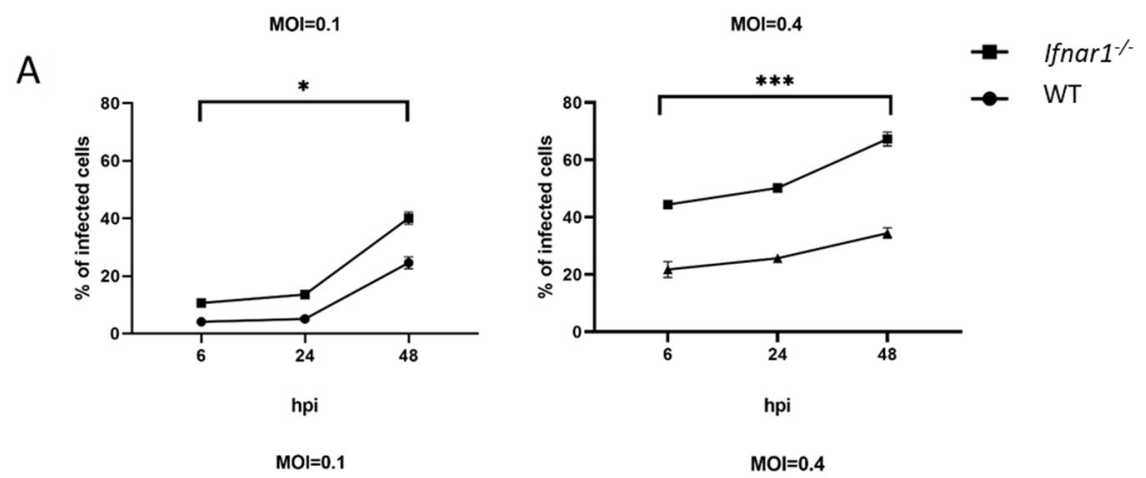

B
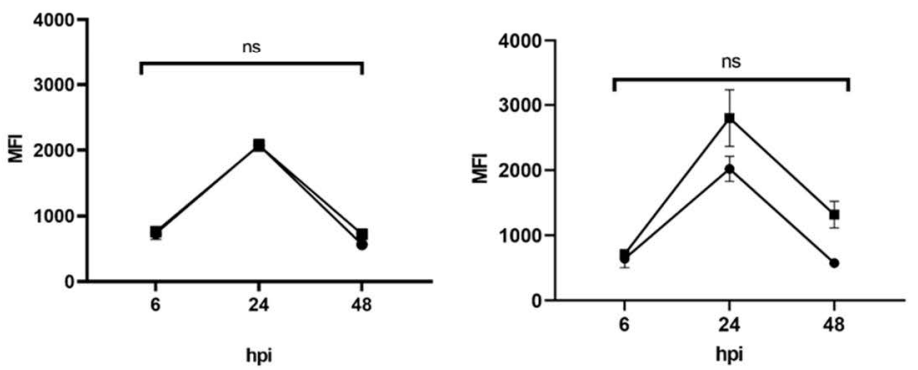

C

$6 \mathrm{~h}$

$24 \mathrm{~h}$

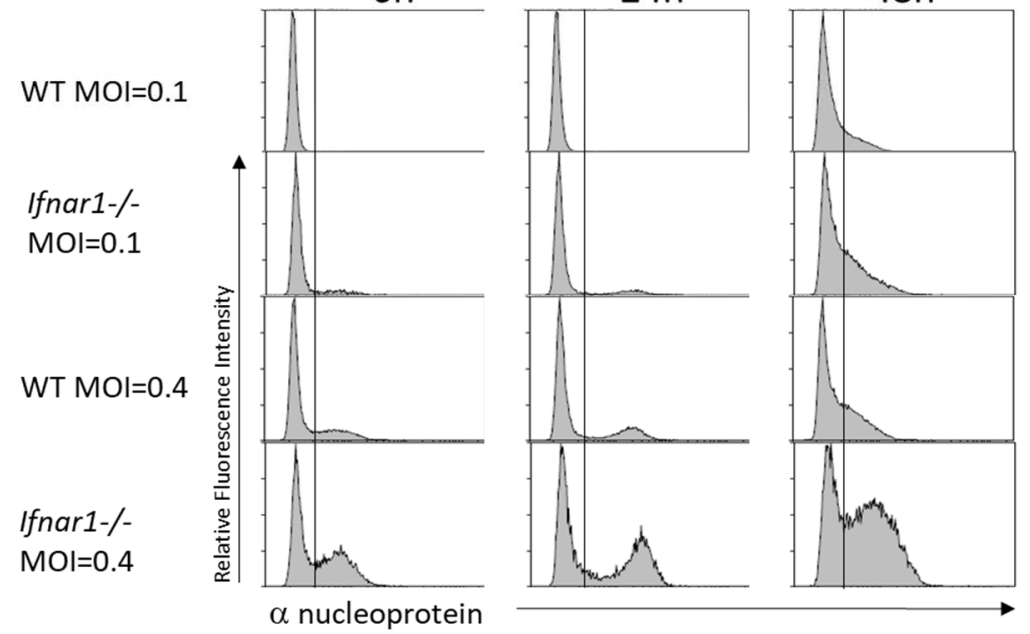

Figure 5. Increases host susceptibility to Influenza virus. (A) Percentage of infected cells determined by FACS by measuring the amount of Influenza NP protein at each given time point. DF1 WT (-•-) and Ifnar1-/- (-口-) cells were infected with Influenza A/WSN/33 (H1N1) at an MOI = 0.1 and MOI $=0.4$ for 6,24 and $48 \mathrm{~h}$. Data were shown as mean $+/-$ SEM $(\mathrm{n}=3)$. Data marked with $\left(^{*}\right)$ indicate $p<0.05$ and ${ }^{* * *}$ ) indicates $p<0.001$. (B) Amount of Mean Fluorescent Intensity (MFI) determined by FACS by measuring the amount of Influenza NP protein inside each DF1 cell at each given time

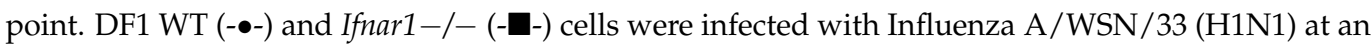
MOI $=0.1$ and MOI $=0.4$ for 6, 24 and $48 \mathrm{~h}$. Data were shown as mean \pm SEM $(n=3)$. Data marked with (ns) correspond to non-significant statistical analyses. (C) Representative FACS histograms of Influenza infected cells determined by FACS by measuring the Relative Fluorescence Intensity of Influenza NP protein at 6, 24 and $48 \mathrm{~h}$ post infection at an MOI $=0.1$ and $\mathrm{MOI}=0.4$.

\subsection{Role of chIFNAR1 in Influenza Virus Infection}

The role of chIFNAR1 in virus infection was further investigated with DF1 WT and chIFNAR1 ${ }^{-/}$cells infected with different influenza virus strains, including WSN, Influenza A/Anhui/1/2013 (H7N9) and HPAI Influenza A/Vietnam/1203/2004 (H5N1). 
At 6 hpi, there was an increased level of NP staining in H7N9 infected chIFNAR1-/cells compared to WT cells, a similar observation as found with WSN (Figure 6). In contrast, significant reduction of HPAI H5N1 infection was found in chIFNAR1 ${ }^{-/}$- cells compared to WT cells (Figure 6). This suggested that different mechanisms were involved in early virus infection when chIFNAR1 ${ }^{-/-}$cells were infected with high and low pathogenicity virus strains.
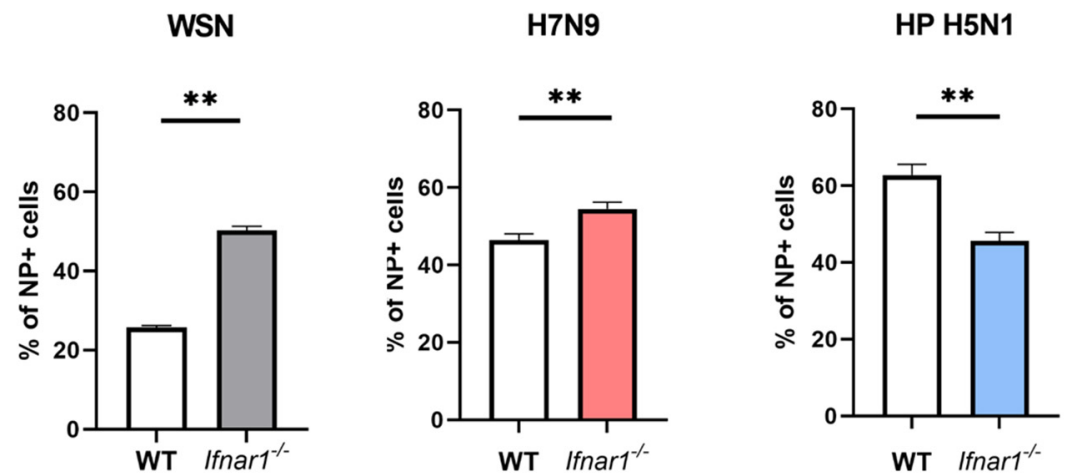

Figure 6. The role of chIfnar1 in influenza infection with different virus strains. DF1 WT and Ifnar1-/cells were infected with WSN (MOI = 0.4), HP H5N1 (MOI = 1), H7N9 (MOI = 1), and viral infections were measured as percentage of NP positive cells at 24 hpi for WSN and 6 hpi for HP H5N1 and H7N9. All data are representative of one independent experiment in triplicate. Data are shown as mean $\pm \mathrm{SD}$ and statistical significance was assessed by unpaired two-tailed Student's $t$-test, ${ }^{* *} p \leq 0.01$. GraphPad Prism 9 software (GraphPad Software, San Diago, CA, USA) was used for statistical analysis.

\section{Discussion}

Continued circulation of $\mathrm{AI}$ viruses in poultry, such as $\mathrm{A}(\mathrm{H} 5)$ and $\mathrm{A}(\mathrm{H} 7)$ viruses, are a public health concern as these viruses cause severe disease in humans. Infections with HPAI virus in gallinaceous poultry leads to rapid onset of severe, systemic disease, often with 100\% mortality [29]. The strategies and control measures used to combat HPAI infection including culling and depopulation, cause severe economic losses to the private and public sectors. Therefore, the knowledge of AI virus infection mechanisms and the host immune responses is crucial to understand the viral pathogenesis in birds and to devise novel strategies against these zoonotic agents. While the IFN pathway and its involvement in the host immune response has been well studied in mammalian species including human and mouse, this pathway in chickens remains unclear. A deeper understanding of IFN pathways and antiviral mechanisms may inform novel therapeutic strategies against influenza viruses that pose major health and economic threats. Thus, the aim of our study was to elucidate the role of IFN signaling in chicken, in particular the chIFNAR1 receptor, in the antiviral response against IAV.

As in mammals, the chicken IFNs bind to the IFNAR receptors to initiate an antiviral response. In this study we compared the amino acidic sequences of chIFNAR1 to the human receptor to predict its tertiary structure. Similar to its human counterpart, chIFNAR1 is composed of 3 domains, a cytosolic domain, a transmembrane domain and an ectodomain which is responsible for the interaction with the ligand [30,31]. Comparison between the tertiary structures of the receptors showed structural homology between the two proteins, suggesting a similar function between the receptors. Phylogenetic analyses of the amino acidic sequence from different mammalian, bird and reptile species indicated evolutionarily conserved properties of chIFNAR1. However, the predicted chIFNAR1 receptor topology needs to be confirmed by more accurate methods such as x-ray crystallography.

We confirmed that the IFN signaling pathway is functional in the DF1 cell line as stimulation of WT cells with IFN $\alpha$ was able to induce a significant upregulation of the chicken $M x$ gene, a previously described ISG. 
To study the role of IFN response during IAV infection we generated a chIfnar ${ }^{-/-}$ DF1 cell line by using CRISPR/Cas9. Molecular characterization of the mutant confirmed the deletion of $97 \mathrm{bp}$ from the first exon of the gene, corresponding to the region targeted by the two sgRNAs. Both the monoallelic and biallelic KO showed a significant reduction of transcription levels. Previous studies have demonstrated, it is critical that both alleles of the gene must permanently be mutated in order to fully inactivate the function of the gene [32]. To further investigate whether the KO of the chIFNAR1 can influence the IFN signal transduction, we measured the transcription levels of some known ISGs such as $M x$ and $P K R$. Our findings show that the transcription levels of $M x$ and $P K R$ are either abolished or strongly reduced in the absence of chIFNAR1, suggesting that IFNAR1 is a key component of the Type I IFN signaling. These results are in accordance with what has been previously observed in $\mathrm{KO}$ mice lacking IFN receptors, which failed to express Mx1 protein and showed enhanced susceptibility to influenza virus [33]. Thus, the activation of $M x$ and PKR in chickens is stimulated by Type I IFNs through activation of the Type I IFN receptor IFNAR1. Cells with an impaired receptor are unable to transduce the IFN signal and activate antiviral effector genes. Intriguingly, our results showed no significant stimulation of $M x$ or PKR expression following IAV infection at either 6 or 48 hpi in WT or chIfnar $1^{-/-}$ cells. The possible explanation is that in vitro virus infection may not induce bioactive IFN and is therefore unable to activate ISG induction. Several studies from different groups also demonstrate that in vitro HP AI virus infection did not induce biologically active Type I IFN in chicken embryo fibroblasts or DF1 cells [34,35]. Viruses may interfere with the IFN response at both the level of IFN synthesis as well as IFN receptor signaling for efficient replication. Indeed, influenza viruses were reported to impair the activation of receptor signaling either by binding viral decoy to Type I IFN to prevent IFN recognition by the receptor, or by degradation of receptors for Type I and Type II IFNs [36], inhibition of JAK/STAT signaling, and regulation of the antiviral function of ISG products [37,38]. Further studies are needed to determine whether influenza virus infection in DF1 cells can induce expression of IFN and a robust IFN response to regulate the antiviral function of ISGs. We were able to demonstrate that a combination of IAV infection and IFN $\alpha$ stimulation were able to induce a strong expression of both ISGs tested. Interestingly, this activation is present still after $48 \mathrm{hpi}$, whereas either IFN $\alpha$ or IAV alone were not able to induce the expression of the ISGs. This is in accordance with previous studies in chicken showing higher expression levels of ISGs including Mx within a few hours post stimulation [39]. In mammals the antiviral role of Mx following IAV infection has been well characterized [14], studies in chicken have reported controversial results on this type of antiviral response. In vivo studies in chicken either did not demonstrate an antiviral effect against H7N1 [18], or showed just a slight reduction in mortality following H5N2 infection [20]. This lack of protection might be due to the lack of GTPase activity shown by chicken Mx protein [19].

Significantly higher virus titers were observed in chIfnar $1^{-/-}$cells at 8 and 12 hpi compared with that of WT cells. This suggests that the anti-viral mechanisms controlled by IFN are limited in the chIfnar $1^{-/-}$cells, allowing more viral particles to be released to the cell culture supernatant. A study from Goodman et al., showed increased influenza viral replication in mouse embryonic fibroblasts lacking the IFN receptor [6]. Recently, Carvajal-Yepes, Monica et al. reported enhanced production of a human influenza virus in IFNAR1-knock-down in immortalized chick-derived PBS-12SF cells than parental cells, supporting the importance of the IFN receptor in controlling viral replication [8]. Lack of IFN signaling also has deleterious consequences for virus infection in H7N9 infected DF1 cells, and our study demonstrated increased levels of H7N9 infection in the absence of IFNAR1 compared to WT cells. In contrast, chIfnar $1^{-/}$cells exhibited decreased levels of infection compared to WT cells, when both cell types were infected with a strain of highly pathogenic H5N1 virus. As demonstrated by other studies, no Type I IFN secretion was observed in chicken DF1 fibroblast cells during highly pathogenic H5N1 infection [40]. We hypothesized that while the IFN receptor is necessary to curb viral replication, there would 
be redundant mechanisms (such as inflammatory response genes) that would be activated as part of the innate immune response upon $\mathrm{HP}$ H5N1 infection, even in the absence of a functional IFN receptor. Further experimentation is needed to interrogate the potential mechanisms of the IFNAR1 response to different viral strain infections. However, the subtype-dependent difference observed in our study offers new insights into the possible roles of IFNAR1 in control and modulation of the infection and replication of different strains of influenza A virus.

Furthermore, virus displayed shorter replication cycle in cells devoid of IFNAR1, with detectable virus level in the supernatant from just $8 \mathrm{hpi}$, in contrast to the detectable virus titers at 12 hpi and onwards in WT cells. Although, the chIfnar $1^{-1-}$ mutant showed higher viral titres at 8 and $12 \mathrm{hpi}$, both cell lines reached similar titers at $24 \mathrm{hpi}$, indicating that there is a maximum (a plateau) number of virions generated within one replication cycle. The higher viral titres observed in chIfnar $1^{-/-}$cells could be because of the lack of early innate immune response in these cells, whereas in WT cells the virus needs more time to disrupt the innate immunity defense and subsequently establish virus infection. Thus, in chIfnar $1^{-/-}$cells the virus is either able to replicate faster and/or it can infect more cells than in WT. To compliment this theory, we used flow cytometry to measure the amount of viral nucleoprotein synthetized during IAV infection in both WT and mutant cells. Our findings confirm what was observed in the previous experiment, that a higher proportion of chIfnar $1^{-/}$cells become infected as compared to WT. Both these results suggest that the IFN response might impact the entry step of the virus cycle, thus the virus is able to access the cells with impaired IFN response more easily than the cells with a normal IFN pathway. Our results indicate that neutralizing the IFN response in cell lines can improve the production of influenza virus and offer a viable alternative system for the production of influenza virus vaccines.

Our overall findings demonstrate that IFNAR1 in chicken is a key component of the IFN transduction signal pathway. It plays a similar role as its human homolog, in its absence, IFN are not able to stimulate the expression of ISGs. Cells with an impaired IFN receptor are more susceptible to the influenza virus infection. Moreover, the lack of chIFNAR1 impacts the entry process of the virus confirming that the innate immunity is a crucial component of the host antiviral defense. We used chicken fibroblast cell line DF1, a homogeneous cell population, for these experiments since they allowed us to study the signaling pathways without immune cell infiltration, which can confound results observed for an animal model. However, it should be stated that by infecting macrophages, dendritic cells, or lung epithelial cells isolated from chickens lacking interferon receptors will enable us to better understand immunity during influenza virus infection. Our findings bring new contributions to the field of host-avian pathogen interaction and innate immunity in chickens. The outcome of our study might have huge implications in the manufacturing of influenza vaccines, as embryonated chicken eggs are the main bioreactors of the seasonal influenza vaccine.

\section{Conclusions}

These studies represent an important finding with regard to the chicken host response to AI virus. We have demonstrated that the role of IFN signaling in response to infection is diverse and can lead to both pro-viral and anti-viral effects. We were able to demonstrate that in the absence of IFNAR1, cells were not only more susceptible to the influenza virus, but also that there was a reduction in replication cycle. Whilst IFNAR1 behaved similarly to mammalian IFNAR1, in that it was able to trigger ISG and reduced viral replication, in the case of $\mathrm{HP} \mathrm{H} 5 \mathrm{~N} 1$ the converse was observed, and viral replication increased. Further investigation into the mechanisms that lead to increased viral replication may provide valuable insights into both the biology of HPAI virus as well as possible means of intervention. 


\begin{abstract}
Author Contributions: Conceptualization, D.S.L. and A.G.D.B.; methodology, K.M., D.S.L., M.D., L.F.M.-O., T.J.G., K.B. and K.A.J.; writing-original draft preparation, K.M.; writing-review and editing, K.M., M.D., D.S.L. and A.G.D.B. All authors have read and agreed to the published version of the manuscript.
\end{abstract}

Funding: This research received no external funding.

Institutional Review Board Statement: Not applicable.

Informed Consent Statement: Not applicable.

Conflicts of Interest: The authors declare no conflict of interest.

\title{
References
}

1. Schoggins, J.W.; Wilson, S.J.; Panis, M.; Murphy, M.Y.; Jones, C.T.; Bieniasz, P.; Rice, C.M. A diverse range of gene products are effectors of the type i interferon antiviral response. Nature 2011, 472, 481-485. [CrossRef] [PubMed]

2. Isaacs, A.; Lindenmann, J. Virus interference. I. The interferon. Proc. R. Soc. Lond. B Biol. Sci. 1957, 147, $258-267$.

3. Santhakumar, D.; Rubbenstroth, D.; Martinez-Sobrido, L.; Munir, M. Avian interferons and their antiviral effectors. Front. Immunol. 2017, 8, 49. [CrossRef] [PubMed]

4. Sick, C.; Schultz, U.; Munster, U.; Meier, J.; Kaspers, B.; Staeheli, P. Promoter structures and differential responses to viral and nonviral inducers of chicken type i interferon genes. J. Biol. Chem. 1998, 273, 9749-9754. [CrossRef] [PubMed]

5. Qu, H.; Yang, L.; Meng, S.; Xu, L.; Bi, Y.; Jia, X.; Li, J.; Sun, L.; Liu, W. The differential antiviral activities of chicken interferon alpha (chifn-alpha) and chifn-beta are related to distinct interferon-stimulated gene expression. PLoS ONE 2013, 8, e59307. [CrossRef]

6. Goodman, A.G.; Zeng, H.; Proll, S.C.; Peng, X.; Cilloniz, C.; Carter, V.S.; Korth, M.J.; Tumpey, T.M.; Katze, M.G. The alpha/beta interferon receptor provides protection against influenza virus replication but is dispensable for inflammatory response signaling. J. Virol. 2010, 84, 2027-2037. [CrossRef]

7. Ng, C.T.; Sullivan, B.M.; Teijaro, J.R.; Lee, A.M.; Welch, M.; Rice, S.; Sheehan, K.C.; Schreiber, R.D.; Oldstone, M.B. Blockade of interferon beta, but not interferon alpha, signaling controls persistent viral infection. Cell Host Microbe 2015, 17, 653-661. [CrossRef]

8. Carvajal-Yepes, M.; Sporer, K.R.; Carter, J.L.; Colvin, C.J.; Coussens, P.M. Enhanced production of human influenza virus in pbs-12sf cells with a reduced interferon response. Hum. Vaccines Immunother. 2015, 11, 2296-2304. [CrossRef] [PubMed]

9. Stanifer, M.L.; Pervolaraki, K.; Boulant, S. Differential regulation of type i and type iii interferon signaling. Int. J. Mol. Sci. 2019, 20, 1445. [CrossRef]

10. Wu, W.; Metcalf, J.P. The role of type i ifns in influenza: Antiviral superheroes or immunopathogenic villains? J. Innate Immun. 2020, 12, 437-447. [CrossRef]

11. Daviet, S.; Van Borm, S.; Habyarimana, A.; Ahanda, M.L.; Morin, V.; Oudin, A.; Van Den Berg, T.; Zoorob, R. Induction of mx and pkr failed to protect chickens from h5n1 infection. Viral Immunol. 2009, 22, 467-472. [CrossRef] [PubMed]

12. Goossens, K.E.; Ward, A.C.; Lowenthal, J.W.; Bean, A.G. Chicken interferons, their receptors and interferon-stimulated genes. Dev. Comp. Immunol. 2013, 41, 370-376. [CrossRef] [PubMed]

13. Haller, O.; Kochs, G. Human mxa protein: An interferon-induced dynamin-like gtpase with broad antiviral activity. J. Interferon. Cytokine Res. 2011, 31, 79-87. [CrossRef]

14. Haller, O.; Kochs, G. Mx genes: Host determinants controlling influenza virus infection and trans-species transmission. Hum. Genet. 2020, 139, 695-705. [CrossRef] [PubMed]

15. Ko, J.H.; Takada, A.; Mitsuhashi, T.; Agui, T.; Watanabe, T. Native antiviral specificity of chicken mx protein depends on amino acid variation at position 631. Anim. Genet. 2004, 35, 119-122. [CrossRef] [PubMed]

16. Li, B.; Fu, D.; Zhang, Y.; Xu, Q.; Ni, L.; Chang, G.; Zheng, M.; Gao, B.; Sun, H.; Chen, G. Partial antiviral activities of the asn631 chicken $\mathrm{mx}$ against newcastle disease virus and vesicular stomatitis virus. Mol. Biol. Rep. 2012, 39, 8415-8424. [CrossRef]

17. Sasaki, K.; Yoneda, A.; Ninomiya, A.; Kawahara, M.; Watanabe, T. Both antiviral activity and intracellular localization of chicken $\mathrm{mx}$ protein depend on a polymorphism at amino acid position 631. Biochem. Biophys. Res. Commun. 2013, 430, 161-166. [CrossRef] [PubMed]

18. Ewald, S.J.; Kapczynski, D.R.; Livant, E.J.; Suarez, D.L.; Ralph, J.; McLeod, S.; Miller, C. Association of mx1 asn631 variant alleles with reductions in morbidity, early mortality, viral shedding, and cytokine responses in chickens infected with a highly pathogenic avian influenza virus. Immunogenetics 2011, 63, 363-375. [CrossRef] [PubMed]

19. Schusser, B.; Reuter, A.; von der Malsburg, A.; Penski, N.; Weigend, S.; Kaspers, B.; Staeheli, P.; Hartle, S. Mx is dispensable for interferon-mediated resistance of chicken cells against influenza a virus. J. Virol. 2011, 85, 8307-8315. [CrossRef]

20. Sironi, L.; Williams, J.L.; Moreno-Martin, A.M.; Ramelli, P.; Stella, A.; Jianlin, H.; Weigend, S.; Lombardi, G.; Cordioli, P.; Mariani, P. Susceptibility of different chicken lines to h7n1 highly pathogenic avian influenza virus and the role of mx gene polymorphism coding amino acid position 631. Virology 2008, 380, 152-156. [CrossRef]

21. Karpala, A.J.; Lowenthal, J.W.; Bean, A.G.D. Identifying innate immune pathways of the chicken may lead to new antiviral therapies. Vet. Immunol. Immunopathol. 2012, 148, 100-109. [CrossRef] [PubMed] 
22. Himly, M.; Foster, D.N.; Bottoli, I.; Iacovoni, J.S.; Vogt, P.K. The df-1 chicken fibroblast cell line: Transformation induced by diverse oncogenes and cell death resulting from infection by avian leukosis viruses. Virology 1998, 248, 295-304. [CrossRef]

23. Reed, L.J.; Muench, H. A simple method of estimating fifty per cent endpoints12. Am. J. Epidemiol. 1938, 27, 493-497. [CrossRef]

24. Kelley, L.A.; Mezulis, S.; Yates, C.M.; Wass, M.N.; Sternberg, M.J. The phyre2 web portal for protein modeling, prediction and analysis. Nat. Protoc. 2015, 10, 845-858. [CrossRef]

25. Karpala, A.J.; Lowenthal, J.W.; Bean, A.G. Activation of the tlr3 pathway regulates ifnbeta production in chickens. Dev. Comp. Immunol. 2008, 32, 435-444. [CrossRef]

26. Stewart, C.R.; Bagnaud-Baule, A.; Karpala, A.J.; Lowther, S.; Mohr, P.G.; Wise, T.G.; Lowenthal, J.W.; Bean, A.G. Toll-like receptor 7 ligands inhibit influenza a infection in chickens. J. Interferon Cytokine Res. 2012, 32, 46-51. [CrossRef] [PubMed]

27. Kochs, G.; Haller, O. Chapter 226-Mx Proteins: High Molecular Weight Gtpases with Antiviral Activity; Academic Press: San Diego, CA, USA, 2010.

28. Goritzka, M.; Durant, L.R.; Pereira, C.; Salek-Ardakani, S.; Openshaw, P.J.; Johansson, C. Alpha/beta interferon receptor signaling amplifies early proinflammatory cytokine production in the lung during respiratory syncytial virus infection. J. Virol. 2014, 88, 6128-6136. [CrossRef] [PubMed]

29. Pantin-Jackwood, M.J.; Swayne, D.E. Pathogenesis and pathobiology of avian influenza virus infection in birds. Rev. Sci. Tech 2009, 28, 113-136. [CrossRef] [PubMed]

30. Uzé, G.; Schreiber, G.; Piehler, J.; Pellegrini, S. The receptor of the type i interferon family. In Interferon: The 50th Anniversary; Pitha, P.M., Ed.; Springer: Berlin/Heidelberg, Germany, 2007; pp. 71-95.

31. Piehler, J.; Thomas, C.; Garcia, K.C.; Schreiber, G. Structural and dynamic determinants of type i interferon receptor assembly and their functional interpretation. Immunol. Rev. 2012, 250, 317-334. [CrossRef]

32. Wang, L.H.; Wu, C.F.; Rajasekaran, N.; Shin, Y.K. Loss of tumor suppressor gene function in human cancer: An overview. Cell. Physiol. Biochem. 2018, 51, 2647-2693. [CrossRef] [PubMed]

33. Mordstein, M.; Kochs, G.; Dumoutier, L.; Renauld, J.C.; Paludan, S.R.; Klucher, K.; Staeheli, P. Interferon-lambda contributes to innate immunity of mice against influenza a virus but not against hepatotropic viruses. PLoS Pathog. 2008, 4, e1000151. [CrossRef]

34. Moulin, H.R.; Liniger, M.; Python, S.; Guzylack-Piriou, L.; Ocana-Macchi, M.; Ruggli, N.; Summerfield, A. High interferon type i responses in the lung, plasma and spleen during highly pathogenic h5n1 infection of chicken. Vet. Res. 2011, 42, 6. [CrossRef] [PubMed]

35. Penski, N.; Hartle, S.; Rubbenstroth, D.; Krohmann, C.; Ruggli, N.; Schusser, B.; Pfann, M.; Reuter, A.; Gohrbandt, S.; Hundt, J.; et al. Highly pathogenic avian influenza viruses do not inhibit interferon synthesis in infected chickens but can override the interferon-induced antiviral state. J. Virol. 2011, 85, 7730-7741.

36. Xia, C.; Wolf, J.J.; Vijayan, M.; Studstill, C.J.; Ma, W.; Hahm, B. Casein kinase 1alpha mediates the degradation of receptors for type $i$ and type ii interferons caused by hemagglutinin of influenza a virus. J. Virol. 2018, 92, e00006-18. [CrossRef]

37. Garcia-Sastre, A. Ten strategies of interferon evasion by viruses. Cell Host Microbe 2017, 22, 176-184. [CrossRef]

38. Pauli, E.K.; Schmolke, M.; Wolff, T.; Viemann, D.; Roth, J.; Bode, J.G.; Ludwig, S. Influenza a virus inhibits type i ifn signaling via nf-kappab-dependent induction of socs-3 expression. PLoS Pathog. 2008, 4, e1000196. [CrossRef]

39. Roll, S.; Hartle, S.; Lutteke, T.; Kaspers, B.; Hartle, S. Tissue and time specific expression pattern of interferon regulated genes in the chicken. BMC Genom. 2017, 18, 264. [CrossRef]

40. Liniger, M.; Moulin, H.R.; Sakoda, Y.; Ruggli, N.; Summerfield, A. Highly pathogenic avian influenza virus h5n1 controls type i ifn induction in chicken macrophage hd-11 cells: A polygenic trait that involves ns1 and the polymerase complex. Virol. J. 2012, 9, 7. [CrossRef] 